\title{
Horizons/Théâtre
}

Revue d'études théâtrales

10-11 | 2017

Genre et arts vivants

\section{Pratiques de résistance en danse actuelle. Husk de George Stamos et Montréal Danse}

\section{Laurane Van Branteghem}

\section{(2) OpenEdition}

1 Journals

\section{Édition électronique}

URL : http://journals.openedition.org/ht/542

DOI : $10.4000 /$ ht.542

ISSN : 2678-5420

\section{Éditeur}

Presses universitaires de Bordeaux

\section{Édition imprimée}

Date de publication : 1 juillet 2017

Pagination : 152-167

ISSN : 2261-4591

\section{Référence électronique}

Laurane Van Branteghem, « Pratiques de résistance en danse actuelle. Husk de George Stamos et Montréal Danse », Horizons/Théâtre [En ligne], 10-11 | 2017, mis en ligne le 01 juillet 2018, consulté le 10 décembre 2020. URL : http://journals.openedition.org/ht/542 ; DOI : https://doi.org/10.4000/ht.542

\section{cc) (1) $\odot$}

La revue Horizons/Théâtre est mise à disposition selon les termes de la Licence Creative Commons Attribution - Pas d'Utilisation Commerciale - Pas de Modification 4.0 International. 


\section{LAURANE VAN BRANTEGHEM}

Libraire, travailleuse culturelle, spectatrice et critique à ses heures, Laurane Van Branteghem est diplômée d'une maitrise en Histoire de l'art de l'Université du Québec à Montréal. Son travail de recherche porte sur le potentiel politique (queer et posthumain) d'œuvres de danse actuelle. Cheffe du Pupitre danse d'Artichaut, la revue des étudiant.e.s en arts de I'UQAM durant plus de deux ans, elle y a développé avec un grand intérêt la critique de spectacle. En outre, son travail à l'Agora de la danse, puis à Tangente, deux diffuseurs de danses contemporaines montréalais, l'a mené à connaître en profondeur la scène locale et à s'intéresser de plus en plus aux différentes pratiques, aux réflexions critiques entourant les arts vivants, ainsi qu'au développement de ceux-ci.

Mail : Lauranevan@hotmail.com

Résumé : Les arts du spectacle ont le potentiel d'être un de ces espaces de résistance, en marge de la « domination sexe/genre normative ». Le corps dansant est alors un parfait support plastique aux interactions entre les déterminismes biologiques, les contraintes socioculturelles et le libre arbitre. Cet article traite spécifiquement du spectacle de danse contemporaine Husk (2012) du chorégraphe montréalais George Stamos en collaboration avec la compagnie Montréal Danse et la rockeuse Jackie Gallant. Cette pièce aborde métaphoriquement, mais sans détour, la performativité des genres, en passant par un éclatement de la figure du corps sexué. S'inscrivant dans un prolongement du travail théorique de Foucault sur le biopouvoir et de la pensée queer sur les pratiques de résistance, la danse, avec le corps comme objet, permet-elle aussi de réinventer le sujet?

Parfois à l'aide de prothèses, de costumes, de postures, de gestuelles et d'expressions faciales et verbales, les interprètes dévoilent des discours sur les normes, les rôles, les identités et les organes sexuels. Une analyse de la pièce Husk selon une perspective queer permet de révéler une réelle exploration artistique des processus de subjectivation en tant que réappropriation politique du sujet.

Mots-cLÉs : danse contemporaine, pensée queer, Husk, résistance, normativité.
AbSTRACT: Performing arts have the potential to be one of those resistance areas on the fringes of the "normative sex / gender domination2". The dancing body is then a perfect plastic vessel for interactions between biological determinism, socio-cultural constraints and free will. This article deals specifically with the contemporary dance performance Husk (2012) from George Stamos, a Montreal choreographer, in collaboration with Montreal Danse and the rock musician Jackie Gallant. This play deals metaphorically but frankly with gender performativity through a bursting of the sexual body figure. As part of an extension of the theoretical work of Foucault on biopower and queer theory on resistance practices, would dance, with the body as an object, also allow to reinvent the subject?

Sometimes using prostheses, sometimes costumes, postures, gestures and facial and verbal expressions, the performers reveal discourses on norms, roles, identities and sexual organs. An analysis of Husk from a queer perspective can address a genuine artistic exploration of subjectivations as a political takeover of the subject.

KEYWORDS: performance, Queer theory, Husk, resistance, normativity. 


\section{Pratiques de résistance en danse actuelle. Husk de George Stamos et Montréal Danse}

Le philosophe, comme l'artiste, n'est pas là pour décrire la réalité, mais pour la transformer ${ }^{3}$.

Paul B. Preciado

\section{Introduction}

Manteau de fourrure vieillot, perruque fade, talons hauts rouges clinquants et postures aguicheuses malhabiles, deux jeunes femmes, assises côte à côte sur leurs chaises, se jettent des coups d'œil de biais et s'imitent l'une l'autre. Elles semblent plutôt inconfortables dans cet accoutrement et les deux mains posées sur un genou, elles accentuent la posture. La cuisse montrée au public, elles agitent un pied chaussé. Elles se recoiffent nonchalamment, s'assurent que leur maquillage ne coule pas. L'une fait pendre sensuellement son soulier au bout de son pied. L'autre l'imite, sans succès, elle doit s'aider de sa main pour décrocher le soulier. Il tombe. Elle le ramasse, le replace et fait mine de l'admirer en étirant la jambe. À partir de leurs mimiques oscillant entre la satire et la caricature, les jeunes femmes révèlent l'importance des stéréotypes de genre et de l'agir qui se conforme aux normes. Tels sont les codes, les canons et standards de l'expression d'un certain genre féminin, semblent-elles nous dire.

Avec une maladresse feinte et une certaine gaucherie, de manière à souligner le jeu de singerie, d'imitation et d'autocritique, Jackie Gallant et Elinor Fueter ouvrent ce spectacle de danse intitulé Husk. Présentée pour la première fois en 2012 à l'Agora de la danse de Montréal, cette collaboration entre la compagnie Montréal Danse, la compositrice, musicienne et performeuse Jackie Gallant et le chorégraphe George Stamos, aborde métaphoriquement, mais sans détour, la performativité des genres ${ }^{4}$. 
En matière de danse contemporaine, le concept « du corps comme construit social genré ${ }^{\prime} \gg$ peut être largement révélé. De nombreux procédés esthétiques employés dans la pièce Husk participent d'ailleurs à créer une confusion des genres et à en imaginer de nouveaux. En maniant l'ambiguïté, le dynamisme et la provocation ${ }^{6}$, des dominantes de son travail, Stamos s'inscrit bel et bien dans une histoire de la danse postmoderne dont les discours (identité, marginalité, binarisme, etc.), les concepts (déconstruction, contre-pratique, détournement, appropriation, etc.) et les modes d'existence singuliers ${ }^{7}$, des modes de vie ou d'être distincts (vulnérabilité, dynamisme, expériences, etc.), font entièrement partie de l'imaginaire actuel de l'artiste. Ils lui permettent ainsi d'ouvrir la porte à certaines formes de résistances et de détournements des processus de subjectivations dominants. Cet article entend montrer que ce spectacle de danse actuelle a le potentiel d'être un de ces espaces de résistance, en marge de la « domination sexe/genre normative ${ }^{8} \gg$.

La danse est certainement un des médiums artistiques où la portée expressive du corps est la plus prégnante. Le corps dansant en représentation possède une forte puissance de persuasion, de rhétorique et de déconstruction ${ }^{9}$. Depuis son émergence autour des années soixante, la danse contemporaine occidentale a permis à de nombreux chorégraphes ${ }^{10}$ d'exprimer différents discours sur la sexualité et sur l'identité sexuelle à travers leurs œuvres. À l'aide de prothèses, de costumes, de postures, de gestuelles ou d'expressions faciales et verbales, les interprètes déconstruisent des discours sur les normes, les rôles, les identités et les organes sexuels. En danse contemporaine, explique le chercheur français Roland Huesca, « l'heure est au bizarre, à une esthétique de la dissemblance et de l'écart entre les représentations normatives du corps humain et l'objet/corps présenté ${ }^{11} \gg$. En effet, la danse permet de réinventer le sujet, mais aussi de révéler les différents procédés et les effets de normalisation par et sur le corps. Par exemple, les postures décrites en ouverture en sont un bel exemple puisqu'elles permettent de réaliser simplement les effets de contraintes artificielles imposés à la performativité du genre féminin. En présentant les gestes ostensiblement genrés, vêtus de costumes connotés (fourrure, talon), les deux personnages féminins adoptent des comportements stéréotypés qui renforcent la proposition avançant que la performativité est de se définir « pour et par l'Autre ${ }^{12}$ », principalement à travers les vêtements, les postures, les comportements et les pratiques de socialisation.

Le corps dansant est alors un parfait support plastique aux interactions entre les déterminismes biologiques, les contraintes socioculturelles externes 
et le libre arbitre. La danse « ouvre nécessairement des espaces et des temps extra-quotidiens où les systèmes normatifs peuvent se trouver paradoxalement mis en échec et où d'autres habitus peuvent se mettre en place et s'incorporer $^{13} \gg$. Car un corps dansant peut incarner explicitement l'imaginaire qu'il désire partager. Où se situe donc Husk à l'intérieur de ces différentes formes d'expressions corporelles ? Cette pièce bouscule-t-elle réellement et brouille-t-elle les codes de l'identité genrée ? Permet-elle une exploration des subjectivations, comme réappropriation politique du sujet?

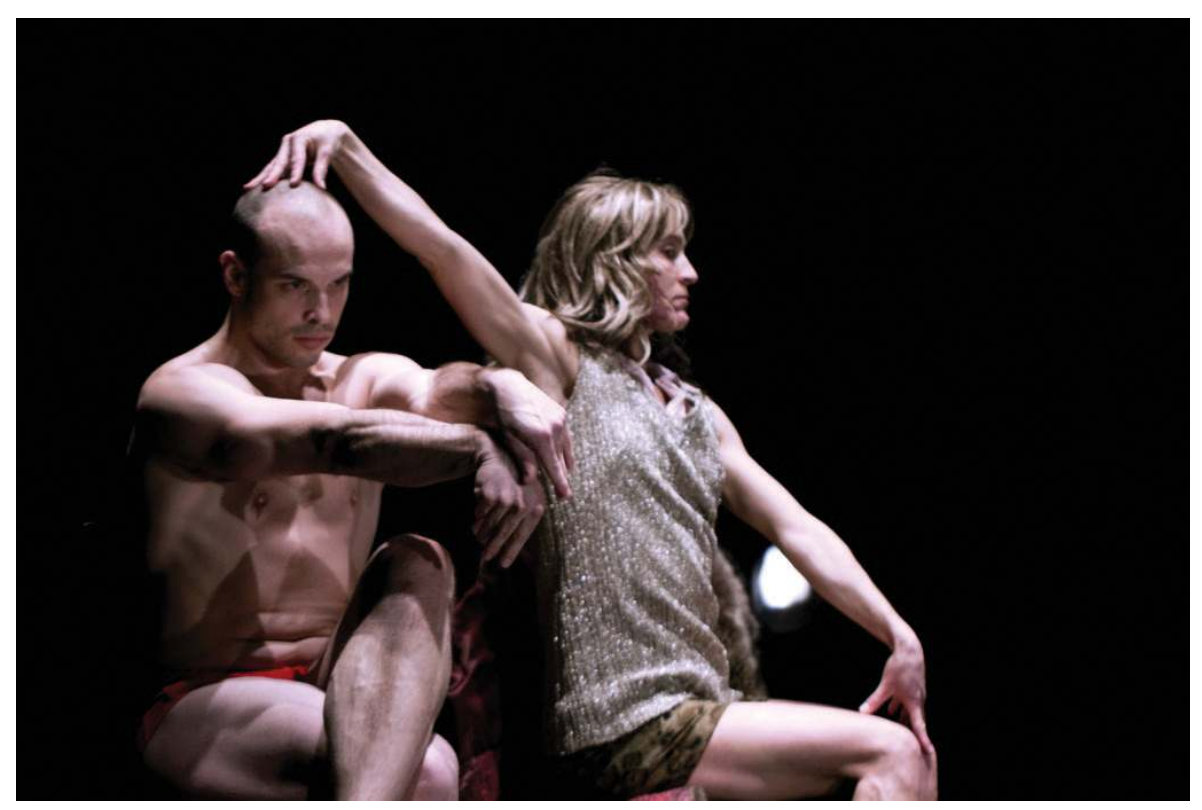

Husk, 2012

Crédit photographique : Alejandro de Leon Interprète : Elinor Fueter et Frédéric Marier

\section{Biopouvoir et corps queer}

Pour comprendre comment certaines pratiques artistiques peuvent résister aux modes de subjectivation dominants qui marginalisent inéluctablement une partie de leurs sujets, il est nécessaire d'introduire et de définir brièvement ce qui est entendu par biopouvoir et ce que la pensée queer introduit. Globalement, celle-ci conçoit différentes formes de résistance aux schémas sociaux, culturels, économiques et politiques dominants, soit principalement 
des résistances aux normes qui rendent possible le plein épanouissement du capitalisme et l'intégration contraignante des corps en son sein.

Les prémisses théoriques des exégèses queer découlent largement de la conception plus générale du pouvoir selon Foucault. C'est sur de nombreux postulats de départ, conceptualisés et énoncés par ce philosophe et historien français dans les années 1970, que s'est érigée la pensée sur le genre, puis la pensée queer dans les années 1990. Historiquement, selon Foucault, d'un pouvoir souverain de répression, l'âge classique amène de nouveaux types de mécanismes de pouvoir. Pour beaucoup de courants théoriques en sciences sociales intéressés par les concepts de déconstruction (postcolonialisme, étude de genre, cultural studies), le pouvoir se conçoit désormais comme une multitude de rapports de force, il devient ainsi positif et producteur, c'està-dire qu'il a pour forme et fonction d'inciter, de renforcer, de contrôler, de surveiller, de majorer et d'organiser les forces qu'il soumet ${ }^{14}: \ll$ le pouvoir s'est donné pour fonction de gérer la vie $\mathrm{e}^{15} \gg$. Nous serions donc dans une ère du biopouvoir où le corps contemporain est manipulé, régulé, calculé, pris en charge et donc normalisé par des formes de pouvoir variées provenant notamment de la famille, de la médecine, de la science, de l'école, etc. Les normes interviennent sur l'intériorité des comportements individuels et collectifs, elles agissent comme des régulateurs de soi. Dans cette optique, le corps devient un élément central dans une réflexion critique sur les processus de normalisation et de subjectivation.

La pensée queer émerge suite à plusieurs vagues de féminismes, en réaction à un essentialisme, bourgeois et blanc, à une conception masculino-centrée de l'homosexualité, à une critique du système identitaire sexuel binaire et plus généralement à un système patriarcal hétéronormé. Elle se définit avant tout par une politisation de la sexualité qui, au vu des travaux de Foucault sur la sexualité, est dorénavant considérée comme « l'ensemble des effets produits dans le corps, les comportements, les rapports sociaux par un certain dispositif relevant d'une technologie politique complexe $\mathrm{e}^{16} \gg$. À cette théorie de la sexualité, les théoriciens et théoriciennes queer ont ajouté la considération plus spécifique des identités sexuelles marginalisées (LGBTQIA $+{ }^{17}$, $\mathrm{BDSM}^{18}$, bref non hétéronormées). L'approche queer ne cherche pas à faciliter l'intégration des $\ll$ anormaux $^{19} \gg$ aux structures dominantes, mais elle se propose plutôt de déconstruire les définitions mêmes de la normativité2 ${ }^{20}$. Des propositions queer vont participer à créer des espaces alternatifs de construc- 
tion identitaire, des modèles de corporéité différents et vont penser et fabriquer de nouveaux cadres d'intelligibilité.

Le spectre de personne ne peut être cerné ou circonscrit au préalable ${ }^{21}$, spécifie le théoricien queer américain David Halperin, puisque les perspectives sur les modes de vie et d'être évoluent et doivent être mouvantes. La pensée ne se donne pas comme objectif un aboutissement, mais plutôt un éternel questionnement, un décentrement constant qui empêcherait tout individu social de se faire incorporer dans la sexopolitique ${ }^{22}$. Quels peuvent donc être ces espaces alternatifs ? Comment des artistes peuvent-ils participer à créer des espaces plus souples, originaux et ouverts ?

\section{Subjectivation et émancipation}

Comme l'écrit Céline Roux, « l'artiste met en jeu son corps comme producteur de signes, entrainant celui-ci à ne pas être un reflet du monde, mais proposant un "être au monde" nécessairement subjectif ${ }^{23} \gg$. Car le corps ne peut pas être appréhendé comme neutre, il est toujours situé ${ }^{24}$. L'historienne de la danse Laurence Louppe insiste sur l'importance de prendre en compte les types de corps incluant leur bagage esthétique, génétique, idéologique ou historique, dans l'analyse et même dans la description des œuvres. Les corps ne sont pas seulement des supports homogènes à un style chorégraphique. La danse « révèle des manières spécifiques de mettre en scène des usages de soi et du monde ${ }^{25} \gg$ élaborant ainsi des processus de subjectivation.

Quelle est donc cette subjectivité dont parlent les auteurs et comment peut-elle se transformer dans la danse ? « [It's] because dance molds the body and its way of moving, it cannot help but propose models of subjectivity in either an affirmative or negative sense. ${ }^{26} \gg$ Mais tout d'abord, que signifie ce concept de subjectivation?

La subjectivation, comme potentiel d'émancipation et donc comme forme de résistance, agit lorsque l'individu ou le groupe s'approprie une capacité d'agir et participe, de façon éclairée et lucide, à une construction sociale de soi. Dès lors, le processus d'émancipation s'accomplit par « la déconstruction des identités et des appartenances préétablies ${ }^{27} \gg$, par une prise de conscience et une déconstruction des rapports de domination, d'exploitation ou d'oppression. L'époque actuelle serait caractérisée par une subjectivation des groupes qui tentent de déconstruire « ces abstractions totalisantes (universalité, ratio- 
nalité, éternité, etc. $)^{28} \gg$. Le sujet est dorénavant considéré comme un sujet politique. Certains sujets vont chercher à se réapproprier les démarches touchant à la définition de leurs propres corps, des images corporelles qui leur sont imposées, des valeurs et des codes prescrits :

Indisciplinaires, ces corps, avec rudesse ou avec sensualité, gravement ou sarcastiquement, s'insurgent contre les convenances corporelles dominantes et inaugurent d'autres possibles. Récalcitrants et insoumis, irrévérencieux et irrespectueux, assumant pleinement leurs différences, ces corps désentravés et hors limites possèdent une force de frappe critique ${ }^{29}$.

L'auteur et l'autrice de l'article De la dimension critique du corps en actes dans l'art contemporain poursuivent en écrivant que les artistes actuels remettent en cause les forces d'aliénation qui pèsent sur les corps. Pour ce faire, l'espace privé peut dorénavant être exposé publiquement comme une forme de dénonciation et une reprise de pouvoir par l'individu.

\section{La pensée queer}

Plusieurs procédés de subjectivation et stratégies de dévoilement des rapports ou des effets de domination entre les sexes ont été identifiés par certain.e.s auteur.trice.s et théoricien.ne.s queer. La plupart visent l'utilisation des codes et des symboles liés à plusieurs binarismes et proposent différentes formes de négociation avec et entre ceux-ci.

En luttant contre un essentialisme, une surdétermination des lieux communs, en exposant les différences de races, de classes, de pratiques sexuelles, de handicaps, les artistes exposent les « anormaux ». L'imaginaire permet d'envisager des possibles, des constructions ou des expériences sociales différentes, des perspectives inatteignables par la rationalité, la productivité ou l'utilité traditionnelle.

Dans un moment fort du spectacle, l'interprète Rachel Harris revêt une prothèse d'un buste qui présente une fausse musculature avec abdominaux et biceps nettement dessinés, et additionnée d'un phallus qui pend mollement dans l'entrejambe. Bien sûr, ces attributs ne sont d'abord qu'un costume, mais la danseuse en prend possession et en réinterprète le sens et l'expressivité par une dynamique et une esthétique singulière. 


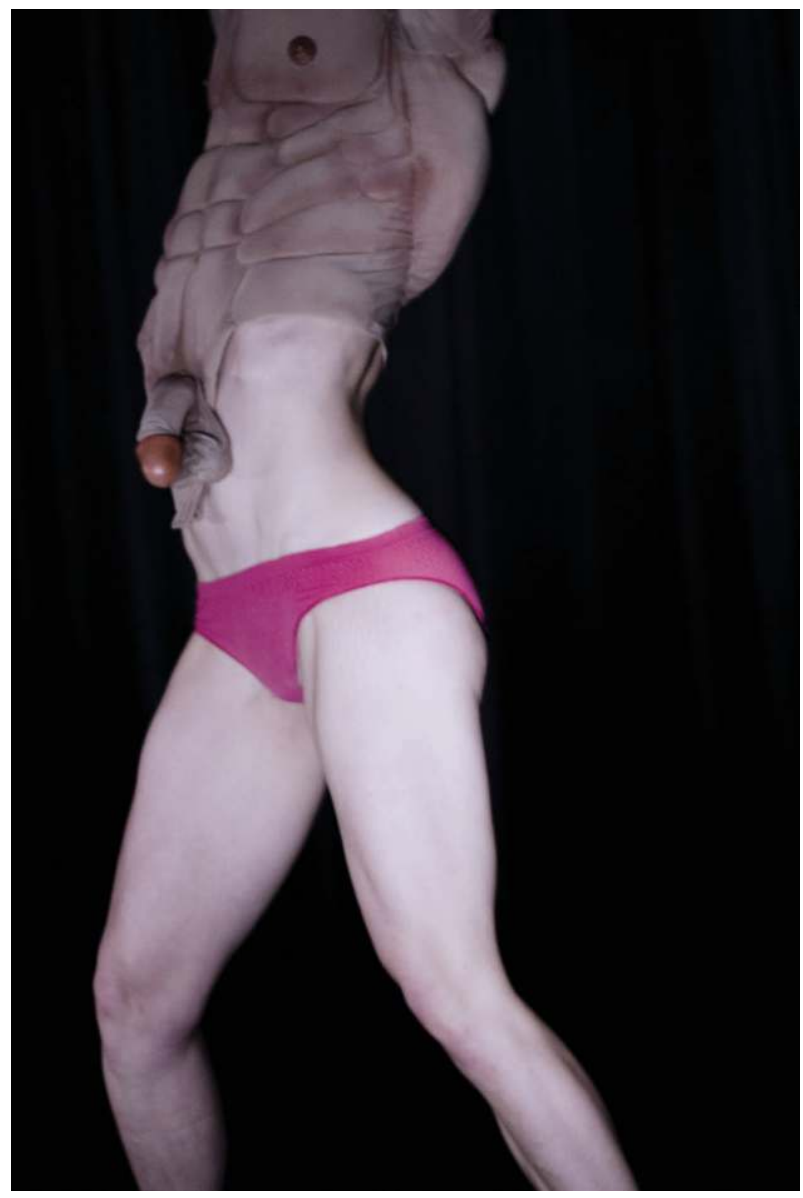

Husk, 2012

Crédit photographique : Alejandro de Leon Interprète : Rachel Harris

D'abord, elle semble prendre le contrôle du corps qu'elle dirige sous la contrainte de sa propre main, elle découvre et explore ses possibilités en étirant chacun de ses membres. Elle prend conscience de l'espace qu'elle occupe, mais aussi de celui qui est disponible. Elle allonge les jambes, les replie, sa gestuelle reste un peu linéaire et rigide. Puis, de dos, les jambes droites et bien écartées, elle se penche et présente ses fesses au public. Elle ne porte sous son costume qu'une culotte rouge. L'appendice qui lui sert de pénis ballote entre ses jambes. L'image est très explicite et provoque un inconfort. Elle n'est pas dans la suggestion, mais montre clairement les organes génitaux masculins. En plus de l'exhibition des organes sexuels, cette présentation est 
quelque peu inhabituelle, le sexe masculin ballote, balance et tangue. Il est rattaché au corps, mais est secoué mollement. Cette image du sexe mâle va à l'encontre d'une représentation virile, forte et brute de la masculinité. Elle maintient toutefois une gestuelle saccadée et tendue évoquant un corps qui ne lui appartient pas complètement. Certaines pauses rappellent l'animalité, comme lorsqu'elle se déplace pieds et poings au sol tel un primate, ou lorsqu'elle réalise des positions sexuelles explicites en s'asseyant sur ses talons et en donnant des coups de bassin vers le haut. Avec son visage fin, ses fesses arrondies, sa puissance musculaire et la précision de ses mouvements, Harris crée un corps qui brouille les genres. En inventant une nouvelle gestuelle qui, malgré des attributs biologiques explicitement masculins, réussit à détourner les conventions essentialistes du genre, la danseuse parvient à « déformer les prescriptions ${ }^{30} \gg$ conventionnelles.

Avec un besoin urgent de sortir de ce costume et un essoufflement évident, Harris achève cette séquence visiblement ébranlée, mais calme et sereine, bien droite, reprenant son souffle et posant une main sur sa hanche, geste de confiance. À travers sa performance, son corps devient hybride dans une cohabitation complexe des deux sexes, glorifiant une culture du corps jusqu'à la confusion.

D'après Bernard Andrieu, en devenant hybride, « notre corps transforme un objet partiel extérieur à lui-même en une partie de son organisation fonctionnelle par des usages subjectifs ${ }^{31} \gg$. Dans une volonté de bouleverser les catégories de genres, cette stratégie s'inscrit entre le travestissement et l'hybridation $^{32}$ puisque les changements sont visibles, exhibés, mais aussi perçus à travers l'interprétation de la danseuse, performés. De plus, par la qualité des mouvements, ce n'est pas seulement l'incohérence des sexes en simple terme de matérialité qui est mise en avant, mais les attitudes conventionnées, les positions corporelles genrées et les gestualités stéréotypées. L'interprète met en évidence le corps et l'utilise comme un espace en construction et de contestation. Usant des procédés de dés-identification et de réappropriation, son corps cesse d'être une matière passive et naturelle où le genre est préinscrit pour devenir un espace qui illustre et dévoile l'acte de performativité.

En entretien, le chorégraphe George Stamos explique que Husk, terme qui veut dire enveloppe, membrane ou coquille, porte sur la relation que chacun nourrit avec son propre corps et comment celui-ci nous définit ${ }^{33}$. Il dit s'être 
inspiré du fait qu'il questionnait déjà son identité sexuelle durant l'enfance. Il place lui-même les questions de genre au centre de son travail. Il explique qu'il fut conscient très tôt des normes et des codes qui régissent la sexualité, du regard des autres, et il affirme être conscient de la performativité de soi, du concept de performativité de l'identité sexuelle.

\section{Performativité}

Le concept de performativité a été introduit par John L. Austin, philosophe du langage, qui distingue l'énoncé constatif, de l'énoncé performatif. L'énonciation performative fait advenir ce qu'elle dit. Cette conception du performatif comme s'autoréalisant en actes, a été reprise et élaborée par Judith Butler dans sa théorie du genre. Selon elle, la sexualité est avant tout une construction sociale. Elle explique que l'individu, en étant témoin des normes sociales, culturelles, politiques, économiques et historiques, est porté à répéter des comportements cohérents vis-à-vis du rôle sexuel qui lui correspond. Ces gestes et comportements sont performatifs. Comme l'écrit si clairement Alexandre Baril, un spécialiste québécois de la pensée de Butler :

En résumé pour Butler, le genre est un ensemble de normes régulatrices orientées téléologiquement vers un idéal de genre, le masculin ou le féminin, qui fait advenir ce qu'il dit, ce qu'il nomme et ce qu'il répète incessamment. [... ] $\mathrm{Ce}$ n'est pas un sujet indépendant qui joue ou qui performe le genre, mais bien que le sujet soit lui-même constitué, c'est-à-dire construit et reconstruit à travers cette performativité du genre. Autrement dit, le genre fait advenir le sujet tel qu'il est $t^{34}$.

Selon Butler, le sexe naturel serait une donnée fluide qui se déploierait en continuum plutôt que binaire, rigide et immuable : masculin, féminin. Le sujet se construit donc à travers cette performativité du genre, cette répétition, cette normalisation.

À ce sujet, S.L. Foster écrit : « Choreography therefore serves as a useful intervention into discussions of materiality and body by focusing on the unspoken, on the bodily gestures and movements that, along with speech, construct gendered identity. ${ }^{35} \gg$ Dans son article « Choreographies of gender $»$, elle avance que l'art chorégraphique dépasse en quelque sorte la performativité du discours pour y inclure celle du mouvement et ainsi permettre de déconstruire un éventail encore plus large de codes et de conventions du genre. 
Revenons par exemple à la scène décrite au tout début de cet article : deux performeuses, assises sur une chaise, singeant une féminité à la limite de la grossièreté. Une de ces deux femmes est Jackie Gallant, chanteuse, musicienne et performeuse, n'ayant jamais suivi de formation professionnelle en danse. Son corps n'est pas conforme à celui musclé, élancé et mince de ses collègues largement entrainés. En plus d'arborer une attitude vaguement narquoise et méfiante, ses gestes sont moins précis et, positionnée à côté d'Elinor Fueter, diplômée de l'école de danse contemporaine de Montréal et interprète pour Montréal Danse depuis 2010, sa gaucherie et son léger manque d'élégance contrastent. La scène est teintée d'humour et c'est avec un regard ironique que le spectateur perçoit ce moment partagé. L'humour, et l'ironie plus spécifiquement, est selon Haraway « une stratégie rhétorique, une méthode politique ${ }^{36} \gg$. L'humour et l'ironie aux services d'une visée spécifique, ici la déconstruction des genres, permettent de révéler le caractère absurde et grossier d'une situation. Il permet de « détourne [r] le lecteur de la signification de surface ${ }^{37} \gg$. La cohérence d'une scène est ébranlée et une nouvelle interprétation du spectateur est sollicitée. Un deuxième degré de lecture apparait qui déstabilise le premier. Le commun peut alors devenir irréel et irrationnel. Dans Husk, « on passe sans cligner des yeux du formel à l'absurde, de la graviteé à la légèreté, de l'essentiel au superficiel, de la supercherie à l'authenticitée ${ }^{38} \gg$.

\section{Danse et résistances}

Au tout début du spectacle, deux interprètes sont couchés face contre sol. Il est impossible de les identifier spécifiquement. Tous deux portent un teeshirt rouge, un pantalon beige et ont une morphologie des plus semblables qui suggère le masculin : un peu forts d'épaule et de buste, avec une taille plus fine. Malgré tout, on discerne un bas de nylon qui cache les cheveux d'une des interprètes pour ressembler le plus littéralement à l'autre dont les cheveux sont rasés. En portant attention, on distingue que ce même interprète présente aussi une forme étrange de musculature grâce à une prothèse portée sous ses vêtements qui évoque celle du corps « viril » créant ainsi l'illusion de similitude avec son partenaire. Le duo reste longtemps en arrière-scène, de dos, et entretient un certain mystère autour des signes distinctifs du genre des interprètes.

Les danseurs se mettent ensuite à bouger avec plus d'ampleur. Leurs gestes sont longs, leurs jambes restent entrouvertes, ils vont jusqu'au bout de leurs 
extensions et gardent continuellement les poings fermés. La gestuelle est de plus en plus stricte, géométrique et rythmée. Petit à petit, une rivalité se dessine entre eux, ils se cognent la poitrine et se poussent, tel un combat de coqs, entravant ainsi leur fluidité. En gardant les bras ouverts, ils dressent leur cage thoracique, intensifiant le rapport de domination qui s'installe. Dans le pas de deux qui s'ensuit, chaque interprète tente de prendre le dessus, puis se retire subtilement pour laisser l'autre diriger l'impulsion.

Ce duo incarne un affrontement qui montre la brutalité sourde, l'orgueil et la domination. Comme l'écrit la critique Frédérique Doyon : « la gestuelle montre sans le mimer que notre image dépend du regard de l'autre : dans ses chaînes de mouvements initiés par un autre partenaire ${ }^{39} \gg$. Le corps de l'un dirige l'autre puis un renversement se produit et l'autre devient le dominé. Husk est truffé de duos mettant en scène différentes relations de domination qui se transforment et se renouvellent à l'intérieur d'une même séquence. Ce niveau de représentation est important puisque plusieurs chorégraphies tendent à réitérer des rapports de pouvoir homme/femme dans leur démonstration traditionnelle de la danse. Les duos sont la majorité du temps mixte, les danseuses sont portées, les danseurs ont une gestuelle plus ample, exécutent plus de sauts, sont plus grands que leur partenaire, etc. Ici, les rapports de forces sont réfléchis pour être non conformistes, tous les danseurs, masculins ou féminins, naviguent entre une gestuelle expansive, rude, énergique et un mouvement de repli, introspectif et plus en retenue ${ }^{40}$. Cette alternance entre deux registres formels peut être comprise comme une métaphore des processus de socialisation, intrinsèques à la formation des identités.

Soudainement, Marier et Harris rompent leur séquence pour s'immobiliser au sol, les jambes étendues, le bras replié sur la hanche et affichant un air efféminé, ils semblent narguer le public du regard. Puis, ils se replacent l'entrejambe d'une main ferme montrant, l'un à la suite de l'autre et sans aucune rupture, deux clichés de genres. Cette superposition rapide et grossière provoque la surprise et rompt momentanément avec les attentes et la norme.

Tout en subtilité et puissamment incarnés, les deux interprètes, en réalité un homme et une femme, questionnent les modes d'être genrés habituels et les relations de domination normatives. Leurs corps amènent une confusion quant aux représentations de genre en proposant aux spectateurs de repenser leurs repères devant ces corps plus difficilement qualifiables et aux comportements parfois insaisissables. 


\section{Conclusions}

Un risque qui plane sur beaucoup de pratiques artistiques qui se considèrent ou sont considérées comme queer est celui des fausses déconstructions. Plutôt que d'ouvrir réellement les représentations politiques grâce à l'imaginaire, à la transformation et à la suggestion des corps, et de déconstruire les modes de subjectivation dominants, plusieurs tendent à la « reproduction de nombreux binarismes straights : privé/public, maître/élèves, homme/femme, etc. ${ }^{41} \gg$, comme le rapporte Frédéric de Carlo et Frédéric Gies dans leur insurrection contre la récupération queer. Néanmoins, Husk parvient généralement à éviter cet écueil en offrant plutôt des propositions esthétiques plurielles qui ne bousculent pas uniquement le savoir traditionnel, mais explorent des constructions de soi créatives et des modelages de soi (littéralement) et non pas seulement un soi emprunté, copié ou en opposition binaire à une identité normée.

De plus, un de ces éléments critiques sensible pourrait être la présence du travestissement comme procédé de déconstruction identitaire. En mettant en scène une représentation visuelle simplifiée d'un certain genre, le travestissement peut être conçu comme renforçant automatiquement le genre représenté. Seulement si nous acceptons aussi le travestissement comme une critique de la conformité, mais surtout de l'essentialisation du genre comme Butler le suggère, les représentations du masculin dans les extraits décrits plus haut ne sont pas particulièrement conventionnelles. Elles n'amplifient pas à outrance les prescriptions du rôle traditionnel masculin, hormis une certaine exagération de la confrontation, l'insistance d'une démonstration de force des interprètes. Les couleurs plutôt neutres des vêtements, les gestes amples, l'ambivalence et le va-et-vient entre une gestuelle plus projetée et agressive et une autre plus souple et sensuelle permettent de concevoir une masculinité multiple qui ne s'identifie pas uniquement en opposition avec les représentations traditionnelles du genre féminin.

Par contre, le sexe (ici compris comme organe sexuel) persiste comme composante centrale dans cette proposition artistique queer, ce qui reconduit un système où le sexe est le principal élément lié au genre. Oui, il y a confusion des genres à travers un ou une même interprète, mais, aucune autre singularité n'est exposée et mise en valeur. Comme l'écrit l'artiste queer Coco Riot : «S'intéresser à la sexualité de manière critique suppose, au-delà du fait de montrer des sexualités qui transgressent les normes morales hétéro- 
normées, de se demander quels corps sont érotisés (plus souvent lesquels ? Jamais quels autres...) et en fonction de quels critères ${ }^{42}$. $\gg$ La scène peut justement être un lieu de représentation public de réalités alternatives.

Rendre visibles des réalités alternatives, les positionner dans l'espace commun, c'est ainsi permettre aux sujets minoritaires de se constituer comme sujets politiques. Parce que son registre demeure dans le cadre symbolique, l'art de la scène permet la mise en place d'un espace de représentation publique qui peut être considéré comme sécuritaire et qui tend à valoriser les modes d'être alternatifs. La « pratique artistique bénéficie d'une marge de tolérance face à ses écarts de conduite. [...] Le théâtre peut être un sanctuaire permissif $^{43} \gg$, explique Frédéric Gravel, chorégraphe et chercheur montréalais. De même, le rassemblement de modalités propres au spectacle fait en sorte que les conditions d'existence soient permises pour laisser la possibilité au sujet d'advenir, d'explorer des modes d'être multiples. À l'intérieur d'une œuvre qui ouvre les possibles et présente des mises en forme visuelle variées, des fantasmes, des délires, chaque sujet peut trouver quelque chose de différent qui lui convient, un élément à ajouter à son propre processus de subjectivation, à l'élaboration de sa définition de soi. L'idée est de permettre d'inclure une plus grande pluralité au sein du « socialement acceptable », de ce qui évolue au même titre que les autres dans l'espace public, de ce qui est valorisé socialement, politiquement, culturellement et économiquement.

\section{Notes}

1. Teresa De Lauretis, " Eccentric Subjects. Feminist Theory and Historical Consciousness », Feminist Studies n 16, 1990, p. 115.

2. Ibid., p. 115 .

3. Claire Grino, «Beatriz Preciado », Inter : art actuel n 112, 2012, p. 24.

4. Au cours de cet article, le genre sera entendu comme " un élément constitutif de rapports sociaux fondés sur des différences perçues entre les sexes, et [...] comme une façon première de signifier des rapports de pouvoir ", Joan Scott et Éléni Varikas, "Genre : Une catégorie utile d'analyse historique », Les Cabiers du GRIF n ${ }^{\circ}$ 37-38, 1988, p. 140.

5. Anne-Marie Dubois, " Les Représentations de figures genderqueers en art actuel. Identités et sexualités : lieux de subjectivités dissidentes ", Mémoire de maitrise présenté à l'Université du Québec à Montréal, Montréal, 2014.

6. George Stamos, Site de George Stamos. Consulté le 8 février 2016. URL : http://www. georgestamos.org/ 
7. Roland Huesca, La Danse des orifices, Paris, Jean-Michel Place, 2015, p. 10.

8. Teresa De Lauretis, « Eccentric Subjects. Feminist Theory and Historical Consciousness », op. cit., p. 115.

9. Mark Franko, «Dance and the Political: States of Exception », Dance Research Journal, vol. 38, no 1, 2006, p. 6.

10. Par exemple le chorégraphe New Yorkais Trajal Harrell et sa pièce Antigone, Dave St-Pierre et La Pornographie des âmes, Cecilia Bengolea et François Chaignaud avec leur spectacle Pâquerette, et bien d'autres.

11. Roland Huesca, Ce que fait Deleuze à la danse, Strasbourg, Le Portique, 2007, p. 5.

12. Anne-Marie Dubois, "Les Représentations de figures genderqueers en art actuel. Identités et sexualités : lieux de subjectivités dissidentes ", op. cit., p. 34.

13. Hélène Marquié, « Le genre en danse », dans Nadia Méhouar-Hertzberg (dir.), Le Genre, effet de mode ou concept pertinent? Bern, Peter Lang, p. 149.

14. Michel Foucault, Histoire de la sexualité. La volonté de savoir, tome I, Paris, Gallimard, 1976, p. 179.

15. Ibid., p. 181.

16. Ibid., p. 168.

17. Lesbienne, gais, bisexuel, transsexuel, queer, intersexuel, assexuel. Le symbole + est simplement ajouté pour les autres formes de sexualité, sexe, genre qui ne seraient pas inclus dans les lettres de cet acronyme.

18. Bondage et discipline, domination et soumission, sadomasochisme.

19. "Multitudes queer, Notes pour une politique des "anormaux" » est le titre d'un texte de Preciado (2003).

20. Comme l'écrit Judith Butler : «Les ontologies fixes sont, depuis bien longtemps, des sites très importants de la violence ", Judith Butler, Humain, inhumain, Le travail critique des normes, trad. Jérôme Vidal et Christine Vivier, Paris, Éditions Amsterdam, 2005, p. 59.

21. David M. Halperin, Saint-Foucault, Paris, Éditions et publications de l'École lacanienne, 2000.

22. «Une des formes dominantes de l'action biopolitique dans le capitalisme contemporain. " Concept nommé par Beatriz Preciado dans "Multitudes queer ", Multitudes $\mathrm{n}^{\circ} 12,2003, \mathrm{p} .17$.

23. Céline Roux, Danse(s) performative(s) : enjeux et développements dans le champ chorégraphique français, 1993-2003, Paris, L'Harmattan, 2007, p. 10.

24. «Situé » en référence au concept de « savoir situé ", concept privilégié par les perspectives féministes, qui remet en question l'élaboration et la persistance des savoirs traditionnels dominants inscrits dans une hiérarchie de pouvoirs et qui réitèrent inévitablement ces rapports de pouvoir, s'opposant ainsi à la neutralité, l'universalisme et l'objectivité et favorisant les savoirs subalternes, minoritaires, marginaux, locaux, bref un pluralisme scientifique. Voir Dona Haraway, « Savoirs situés », Des singes, des cyborgs et des femmes, trad. Oristelle Bonis, Paris, Éditions Jacqueline Chambon, 2009.

25. Roland Huesca, «Danse. Les différents corps de la technique », Quant à la Danse n 2, 2005, p. 30.

26. «Parce que la danse façonne le corps et sa façon de bouger, elle ne peut échapper au fait de proposer des modèles de subjectivité positifs ou négatifs. " Notre traduction. 
Mark Franko, «Dance and the political: states of exception », Dance Research Journal $\mathrm{n}^{\circ} 1,2006$, p. 7.

27. Hubert Gendron-Blais, «Dimensions politiques de l'émancipation à travers les théories esthétiques et des pratiques artistiques », Montréal, mémoire de maîtrise en science politique, Université du Québec à Montréal, 2012, p. 21.

28. Ibid., p. 24.

29. Jean-Marc Lachaud et Claire Lahuerta, « De la dimension critique du corps en actes dans l'art contemporain ", Actuel Marx n 41, 2007, p. 87.

30. Hélène Marquié, Non, la danse n’est pas un truc de filles!, Toulouse, De L'attribut, 2016, p. 107.

31. Bernard Andrieu, «L'hybridation est-elle normale ? ", Chimères n 75, 2011, p. 22.

32. L'hybridation est ici conçue comme un croisement, un métissage, un mélange de genres, d'espèces ou d'éléments qui ne se mêleraient pas «naturellement » pour créer une entité nouvelle. Selon Andrieu, « elle implique une mobilité et un métissage, engage le corps dans l'altérité par l'altération que lui procure toute incorporation de son environnement, et reconfigure lêtre dans un devenir plastique lié à son adaptation vivante à de nouvelles normes ", ibid., p. 17.

33. News Desk, «Montreal dancer and former sex-worker George Stamos puts the human body on the line - again », Montréal Gazette. Consulté le 7 février 2012. URL : http:// montrealgazette.com/author/montrealgazette

34. Audrey Baril, "Judith Butler et le féminisme postmoderne : analyse théorique et conceptuelle d'un courant controversé ", Sherbrooke, mémoire de maîtrise en philosophie, Université de Sherbrooke, 2005, p. 82. L'auteur se nomme aujourd'hui Alexandre Baril.

35. "La chorégraphie s'avère un outil analytique riche et utile dans les discussions sur la matérialité et le corps, car elle se concentre sur les non-dits, les gestes et les mouvements corporels qui, avec la parole, construisent l'identité sexuelle. » Notre traduction. Susan Leigh Foster, « Choreographies of gender », Signs n 1, 1998, p. 5.

36. Ibid., p. 30.

37. Linda Hutcheon, «Ironie et parodie : stratégie et structure », Poétique : Revue de Théorie et d'Analyse Littéraires n' 36, 1978, p. 469.

38. Montréal Danse et Art circulation, Husk, Dossier de presse, 2014, p. 3.

39. Frédérique Doyon, «Détournement de corps », Le Devoir, Montréal, 9 février 2012.

40. Hélène Marquié, Non, la danse n’est pas un truc de filles !, op.cit.

41. Frédéric de Carlo et Frédéric Gies, «Linvisibilité des queer à b-visible: La récupération du queer ", NDD, 2003, p. 15.

42. Coco Riot, «L'art queer face au sexe », Inter : art actuel n 112, 2012, p. 20.

43. Frédéric Gravel, Le Rôle de l'artiste dans la société démocratique, Mémoire de maitrise présenté à l'Université du Québec à Montréal, Montréal, 2010, p. 132. 